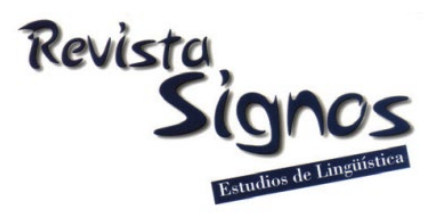

\title{
La función de Tema en español: Sus medios de realización desde la perspectiva trinocular de la Lingüística Sistémico-Funcional
}

\section{The function of Theme in Spanish: Means of realization from the trinocular perspective of Systemic Functional Linguistics}

\author{
Estela Inés Moyano \\ INSTITUTO DEL DESARROLLO HUMANO \\ UNIVERSIDAD NACIONAL DE GENERAL SARMIENTO \\ ARGENTINA \\ estelaimoyano@gmail.com \\ emoyano@campus.ungs.edu.ar
}

Recibido: 08-V-2020 / Aceptado: 05-V-2021

DOI: $10.4067 /$ S0718-09342021000200487

\section{Resumen}

En el presente trabajo se aborda la función de Tema de la cláusula en español desde la perspectiva de la lingüística sistémico-funcional (LSF). Esta función se encuentra comprendida en la metafunción textual y en el estrato léxico-gramatical. Es la función más destacada del sistema de TEMA, de acuerdo con las definiciones de Halliday ([1979] 2002). Particularmente, el objetivo de este trabajo es establecer el medio por el cual la función de Tema se realiza, es decir, se encodifica en la cláusula en español. Esta cuestión ha generado controversias en la bibliografía (por ejemplo, Arús, 2010, 2017; Lavid, Arús \& Zamorano, 2010 vs. Moyano, 2016). Por esta razón, resulta de sumo interés establecer los fundamentos teóricos que orientan la definición de la posición que aquí se asume, así como ofrecer algunos elementos que permiten, mediante el análisis de textos, darle sustento. Con esa finalidad, se establece una interpretación de las funciones de Tema y Nuevo basada en bibliografía clásica en este marco teórico, se propone la aplicación de la metodología trinocular sostenida por Caffarel, Martin y Matthiessen (2004a) y Martin, Quiroz, Wang y Zhou (en prensa) para la descripción de las funciones en juego y se analizan ocho textos para concluir que la función de Tema en la cláusula en español se realiza por dos medios: el Tema no marcado por concordancia con el sufijo verbal, mientras que el Tema marcado lo hace por posición. Se presenta a partir de todo este desarrollo una red sistémica para la función de Tema en español.

Palabras Clave: Lingüística Sistémico-Funcional, tipología lingüística, metafunción textual, Tema, Español. 


\begin{abstract}
The present paper will treat the function of Theme in the Spanish clause from a systemic functional perspective. This function is part of the textual metafunction, located in the lexico-grammatical stratum. It is the most relevant function of the system of THEME, according to the definitions by Halliday ([1979] 2002). The main goal of the paper is to establish the means by which this function is realised in Spanish. This issue is controversial (e.g. Arús, 2010, 2017; Lavid, Arús \& Zamorano, 2010 vs. Moyano, 2016), so that it is relevant to clarify the theoretical elements that orient the position sustained and to substantiate this position through text analysis. To do this, the paper states an interpretation of the functions of Theme and New based in SFL classic literature. Then, proposes a trinocular methodology to orient the description of these functions in Spanish (Caffarel, Martin \& Matthiessen, 2004a; Martin, Quiroz, Wang \& Zhou, in press). Eight texts are analysed in order to conclude that the unmarked Theme in Spanish is realized by agreement with the verbal affix while the marked Theme by position. Finally, a system network will be drawn and explained before the final reflections.
\end{abstract}

Key Words: Systemic Functional Linguistics, language typology, textual metafunction, Theme, Spanish.

\title{
INTRODUCCIÓN
}

En el contexto teórico de la lingüística sistémico-funcional (en adelante LSF), la función de Tema de la cláusula se encuentra comprendida en la metafunción textual y en el estrato léxico-gramatical. Es la función más destacada del sistema de tema, de acuerdo con Halliday, e interactúa con la función que este autor considera más relevante en el sistema de información, el Nuevo, a fin de crear textura (Halliday, [1979] 2002). Esta interacción tiene consecuencias no solamente en el nivel de la cláusula sino del texto, por lo que esta cuestión no puede ser trabajada observando cláusulas aisladas sino desde una perspectiva que, como se verá más adelante, involucra el estrato semántico-discursivo tanto como otros rangos de la léxicogramática y el estrato fono-gráfico.

En su introducción a Language Typology: A functional perspective, Caffarel et al. (2004a) precisamente orientan el trabajo para la descripción de la metafunción textual en una lengua determinada sugiriendo interrogarse acerca de si existen en ella recursos en la sucesión de cláusulas que desarrollen un ángulo [del campo] sobre el cual se esté hablando y si es la función de Tema la que hace esa contribución. De existir esa función, la siguiente pregunta, según estos autores, es cómo se realiza en las cláusulas y, finalmente, si puede distinguirse entre Tema no marcado y Tema marcado en la lengua en cuestión. Asimismo, sugieren preguntarse por la función de Nuevo y su capacidad de construir un punto en el texto y por el medio de realización de esta función. En este conjunto de orientaciones, surge también la pregunta acerca de si existen en esa lengua modos de combinación entre las funciones de Tema y Nuevo en las cláusulas. Estas preguntas, entonces, sugieren un movimiento en el análisis que va 
desde el discurso hacia la cláusula, en consonancia con lo que proponen Matthiessen y Halliday (2009), según se desarrollará más adelante.

El objetivo del presente trabajo es establecer el medio por el cual la función de Tema se realiza, es decir, se encodifica, en la cláusula en español. Esta cuestión ha generado controversias en la bibliografía (por ejemplo, Arús, 2010, 2017; Lavid et al., 2010 vs. Moyano, 2016), lo que hace que resulte de sumo interés establecer los fundamentos teóricos que orientan la definición de la posición que aquí se asume así como ofrecer algunos elementos que permiten, mediante el análisis de textos, darle sustento.

En consecuencia, para lograr el objetivo propuesto, en primer lugar se realiza el planteo de la problemática, que presenta y contrapone las distintas posiciones existentes en la bibliografía sobre Tema en español. En segundo lugar, se presentan los principales fundamentos de la tipología lingüística en el marco de la LSF, teniendo en cuenta bibliografía clásica sobre esta cuestión (Martin, 1983; Rose, 2001; Matthiessen, 2004; Caffarel et al., 2004a) y otra más actualizada (Martin et al., en prensa). A continuación, se aborda el recorte del marco teórico que sustenta la propuesta, basado fundamentalmente en la definición de la función de Tema y, de manera obligada, de su complemento, la función de Nuevo. Se plantea no solo el significado que estas funciones construyen sino los medios de realización que se observan en diferentes lenguas (Halliday, [1979] 2002, 1994; Fries, 1981; Martin, 1992a, 1992b; Matthiessen, 2004; Caffarel, Martin \& Matthiessen, 2004b; Halliday \& Matthiessen, 2014). Seguidamente, a partir de este breve recorrido, se establecen los fundamentos para la metodología utilizada para la descripción que se lleva a cabo. Finalmente, se desarrolla la argumentación que permite sostener que la función de Tema en la cláusula en español se realiza por dos medios: el Tema no marcado por concordancia con el sufijo verbal, mientras que el Tema marcado, por posición. A partir de este trabajo, se presenta una red sistémica para la función de Tema en español.

\section{Marco Teórico}

\subsection{El problema de la realización de Tema en la cláusula en español}

El estudio del Tema en español tiene larga trayectoria. Diversos autores han abordado la cuestión con un objetivo descriptivo (Taboada, 1995; McCabe, 1999; Montemayor-Borsinger, 2009; Arús, 2010; Lavid et al., 2010; Gutiérrez, 2011) y coinciden, según sintetiza muy claramente Arús (2017), en que el Tema en español se realiza mediante el primer elemento experiencial de la cláusula. Este elemento puede ser un participante realizado por un grupo nominal, un proceso, entendiendo en este caso que es relevante el sufijo verbal, que expresa número y persona del verbo 
(Taboada, 1995; Lavid et al., 2010), pero también un pronombre clítico, entendido también como participante, es decir, ubicado en el rango de la cláusula. Gutiérrez (2011) propone, por un lado, que la extensión del Tema en español contiene todo elemento en primera posición en la cláusula hasta el proceso, incluyéndolo. Sin embargo, señala que solo pueden ser Tema un participante (incluyendo los clíticos) o una circunstancia, ya que en el caso de que el proceso sea el primer elemento el Tema estará realizado por el sufijo verbal, como participante.

La propuesta de estos autores surge de considerar, mayormente, la definición de Tema como 'el punto de partida de la cláusula', es decir, de tomar la posición como medio de realización (Taboada, 1995; Montemayor-Borsinger, 2009; Arús, 2010; Gutiérrez, 2011), tal como ocurre en inglés, según la definición de Halliday (Halliday, 1994; Halliday \& Matthiessen, 2014).

Otros autores (por ejemplo, Moya Guijarro, 2003; Filice, 2010; Pérez de Cabrera, 2012; Gómez González \& García Varela, 2014) han tomado alguna de estas descripciones para aplicarla al análisis de textos que instancian diferentes géneros. Algunos de esos trabajos son comparativos con el inglés.

Sin embargo, Moyano (2016) propone que el medio de realización del Tema en español es diferente del que utiliza el inglés. El Tema en español, señala, no se realiza por posición sino por concordancia: el Tema experiencial es siempre un participante que concuerda con el sufijo verbal en número y persona. Esta posición es adoptada por Martin et al. (en prensa). En este trabajo se volverá sobre ello, a fin de reflexionar sobre aspectos teóricos y metodológicos que permiten arribar a esta conclusión así como mediante el análisis de textos de diferentes registros en esta lengua.

\subsection{Fundamentos de la tipología lingüística en el marco de la LSF}

Antes de abordar la cuestión que este artículo se propone dirimir, es necesario presentar muy brevemente algunos fundamentos de la tipología lingüística en el marco de la LSF, a fin de sentar las bases sobre las cuales se desarrolla el trabajo de descripción.

De acuerdo con Caffarel et al. (2004a), la LSF propone la existencia de dos niveles de análisis. El primero es el de una teoría general del lenguaje como sistema semiótico que -cabe recordar- se encuentra en relación de realización con otro sistema semiótico, el contexto. Este nivel teórico distingue categorías universales, las que se proponen como extendidas a todas las lenguas y que comprenden fundamentalmente las cinco dimensiones que abordan Matthiessen y Halliday (2009). La primera dimensión es la estratificación. El lenguage es interpretado como organizado en tres estratos, que constituyen tres diferentes niveles de abstracción en relación de realización: el estrato semántico-discursivo, el léxico-gramatical y el fono-gráfico. La segunda dimensión es 
la metafunción. El lenguaje construye tres tipos diferentes de significados o metafunciones: la ideacional, que permite construir mundo; la interpersonal, que construye relaciones y la textual, que organiza el flujo de información en el texto. La tercera dimensión es el eje. Los tres tipos de significados que genera el lenguaje se organizan en sistemas de opciones, conjuntos de rasgos en relación paradigmática, inferidos de las estructuras que los realizan en textos reales. La cuarta dimensión es el rango, es decir, las diferentes unidades composicionales que se organizan en jerarquías a lo largo de los cuales los recursos sistémicos pueden ser distribuidos, que permiten recursión en la realización. Finalmente, la quinta dimensión es la instanciación, que explica la relación entre el sistema lingüístico completo y los textos como artefactos semióticos (instancias) que se producen a partir de las elecciones que el hablante hace del sistema, elecciones mediadas por el registro y el género, estratos del contexto.

El segundo nivel de análisis tiene relación con la descripción, que aborda el modo en que las diferentes funciones identificadas en la teoría general se ponen de manifiesto en una lengua y a partir de qué recursos. En este sentido, la teoría considera la existencia de rasgos particulares en las diferentes lenguas que merecen ser estudiadas. La implicación más inmediata de la existencia de este nivel es que existen variaciones entre lenguas en relación con su estructura y su comportamiento funcional (Caffarel et al., 2004b; Martin et al., en prensa).

Para dar cuenta de la descripción de una lengua, es necesario abordar las diferentes funciones en relación con las dimensiones teóricas fundamentales. En primer lugar, se necesita ubicar el sistema lingǘstico a describir en un estrato y una metafunción. Luego, es imprescindible diferenciar entre el tipo de significados que un sistema construye y los medios que la lengua bajo descripción utiliza para su realización a lo largo de la escala de rango, es decir, considerando no solamente el nivel de la cláusula sino también los rangos inferiores que pueden intervenir en la realización de la función que se estudia (Martin, 1983; Caffarel et al., 2004a; Martin et al., en prensa).

Establecidos estos puntos de partida, al abordar la descripción es necesario observar el fenómeno a partir de tres perspectivas o perspectiva trinocular: desde arriba, es decir considerando los significados del contexto y del discurso con los que la función en estudio se relaciona en términos de realización; por alrededor, lo que significa tener en cuenta otras funciones que se relacionan en el mismo nivel de análisis con la función objeto de descripción; desde abajo, es decir, la vinculación de la función con elementos de rangos inferiores dentro de la misma metafunción así como la participación de recursos del estrato fono-gráfico, si la hubiera. Este trabajo, en consecuencia, no puede ser llevado a cabo a partir de cláusulas aisladas, sino que es necesario considerar muestras de discurso natural, es decir, porciones de texto. Las descripciones solo son posibles como interpretación de patrones que emergen del 
discurso en diferentes registros (Martin, 1983; Caffarel et al., 2004a; Martin et al., en prensa).

Como paso siguiente, es necesario construir la red sistémica correspondiente a la función analizada, dado que la LSF prioriza por sobre el estudio de las estructuras su relación con el sistema (Martin, 2013; Martin et al., en prensa). Este paso es importante, por un lado, para sistematizar los hallazgos e ir construyendo el sistema general de una lengua. Pero también permite la comparación entre lenguas, otro aspecto fundamental del proceso de descripción y que permite hacer generalizaciones (Matthiessen, 2004).

Finalmente, es imprescindible testear la red diseñada en el análisis de textos de diferentes géneros y registros, a fin de comprobar su validez.

\subsection{Las funciones de Tema y Nuevo en el marco de la LSF: Significado y medios de realización}

Una de las causas de la controversia acerca de la realización del Tema en español se vincula con la interpretación que se hace en cada caso de la definición de Halliday sobre esta función y, concomitantemente, con la discusión acerca de los medios a través de los cuales esta función se manifiesta en diferentes lenguas. Dicho en otros términos, se plantea la cuestión acerca de cuál es el significado que la función de Tema construye en una lengua -es decir un aspecto de la teoría- y cuál es el recurso que diferentes lenguas utilizan para realizar ese significado -aspecto que se relaciona con la descripción.

Halliday señala que Tema es "el elemento que sirve como punto de partida del mensaje; aquello de lo que se trata la cláusula"1 (Halliday, 1994: 37). Esta definición suscitó muchos debates, especialmente en lo que hace a su segunda parte, de lo que nos ocuparemos más adelante. Sin embargo, un aspecto que interesa discutir en primer lugar es la primera parte de la definición, la que considera Tema como 'punto de partida del mensaje'.

Esta formulación ha sido interpretada como la parte inicial de la cláusula, su primer elemento experiencial. Sin embargo, es el mismo Halliday (1994: 38) quien alerta acerca de que "la primera posición en la cláusula no es lo que define el Tema; es el medio por el cual la función de Tema es realizada en la gramática del inglés". E inmediatamente afirma que otras lenguas que tienen una función similar a la de Tema en inglés la manifiestan de otra manera. Efectivamente, Halliday y Matthiessen (2014), si bien mantienen esta primera parte de la definición, reconocen que existen diferentes medios de realización para esta función: la posición en inglés y en algunas otras lenguas, y la segmental o anunciada por medio de una partícula, como por ejemplo en japonés. 
Estos diferentes medios de realización (Caffarel et al., 2004a para el inglés; Martin, 2004 para el tagalog; Teruya, 2004 para el japonés) conducen a Martin (1992a) a proponer que es necesario reflexionar acerca del significado de esta primera parte de la definición de Halliday: qué significa que Tema es 'el punto de partida del mensaje'. Con esta finalidad, se basa en el trabajo de Fries (1981) para señalar que la definición de Halliday significa más que lo que aparece primero'. Para ello, propone que Fries (1981) aporta una interpretación muy reveladora: que la elección del elemento que funciona como Tema en los términos de Halliday tiene motivaciones discursivas. En efecto, Fries (1981) propone que la información provista por los Temas experienciales de las diferentes cláusulas de un párrafo construyen su 'método de desarrollo'. Y esto lo hace eligiendo elementos de una cadena léxica y/o referencial como Temas en las cláusulas sucesivas de ese párrafo. De este modo, el método de desarrollo tiene la función en el discurso de referir a un campo semántico hacia el cual el lector u oyente es orientado.

A partir de allí, Martin reformula la idea de que Tema es "el punto de partida del mensaje" (Halliday, 1994: 37): es la función que orienta a los lectores u oyentes a un ángulo determinado del campo (Martin, 1992a). En este sentido, afirma que la primera posición en inglés "construye un ángulo particular de interpretación en la temática (topic ${ }^{2}$ ) de cada texto, que resuena con otros aspectos de la organización del discurso" (Martin, 1992a: 154).

Así, el método de desarrollo de una fase o un párrafo del texto se construye mediante el conjunto de Temas de las cláusulas de esos segmentos de texto que iluminan un ángulo del campo determinado, fijan la mirada del lector u oyente, anclan el texto en un aspecto del campo, independiente de cómo se realice el Tema en una cierta lengua.

Con respecto a esto, Bartlett y O’Grady (2019) señalan que estas afirmaciones no pueden ser aplicadas a otras lenguas diferentes del inglés: consideran que el método de desarrollo es un recurso específico de esa lengua. Sin embargo, Moyano (2016) ha mostrado mediante el análisis de textos que este patrón se manifiesta claramente en español, cuestión sobre la que se volverá más adelante en este artículo con otros ejemplos. Por otra parte, Rose (2001) ha identificado, como generalización a partir de varias lenguas, que el hablante elige un elemento integrante de una cadena léxica como Tema de la cláusula, y que los Temas de las demás cláusulas de la misma fase pertenecen a la misma cadena léxica, que en conjunto realizan el método de desarrollo de esa fase.

De esta manera, más allá de cuál sea el medio de realización del Tema en las diferentes lenguas (posicional o segmental), importa señalar que, visto desde una perspectiva discursiva (desde arriba), el conjunto de Temas de un fragmento de texto construye el método de desarrollo de ese fragmento. Es decir, el Tema contribuye al 
flujo de información de un texto orientando al lector u oyente a un ángulo del campo. El 'punto de partida', entonces, tiene que ver con esa orientación, no con el medio de realización del Tema de la cláusula. En inglés, el Tema se realiza por el primer elemento de la cláusula; en otras lenguas, se manifiesta de otras maneras. En tagalog, por ejemplo, de manera segmental, mediante un grupo nominal que en su caso no marcado se ubica al final de la cláusula y está señalado por una partícula (Martin, 2004).

Otro aspecto de la definición de Tema mencionada más arriba merece ser tratada: la segunda parte, que señala que Tema es "... aquello de lo que se trata la cláusula" (Halliday, 1994: 37). En el mismo sentido, es necesario tener en cuenta otras definiciones de Halliday ([1979] 2002: 207) que refieren que Tema es "aquello acerca de lo cual el hablante trata" o "la señal de interés del hablante".

Estas definiciones han llevado a la interpretación de que Halliday se refiere al concepto de 'tópico' (Downing, 1991; Thompson \& Thompson, 2009). Sin embargo, el propio Halliday ([1979] 2002) afirma que el significado de Tema es estrictamente textual, no ideacional. Esta función interactúa con significados experienciales e interpersonales para organizarlos en la cláusula a fin de construir patrones de significados en el texto, organizando el flujo de información (Martin, 1996; Martin \& Rose, 2007).

Siguiendo a Halliday ([1979] 2002), Martin (1996) propone que las funciones de Tema y de Nuevo -las dos funciones consideradas relevantes para la construcción de textura- no tienen ningún otro significado que el rol que juegan en la contextualización de un texto:

"El Tema tiene significado con respecto a un patrón de Temas; el Nuevo tiene significado con respecto a un patrón de Nuevos; Tema y Nuevo tienen significado en la relación complementaria entre ambos, como parte de estos patrones complementarios" (Martin, 1996: 48-49).

En esto consiste la función 'habilitadora', que Halliday atribuye a la metafunción textual, como Forey y Sampson (2017) subrayan: la metafunción textual es realizada por elecciones que combinan los recursos para construir realidad y relaciones. Esto refuerza la idea de que la función de Tema experiencial o ideacional es una función textual que levanta material ideacional de la cláusula para crear un patrón que oriente a los lectores u oyentes hacia el campo del discurso, es decir, hacia un determinado aspecto del contexto. En esto coincide la definición de Halliday y Matthiessen (2014: 89) que señala que Tema es "[...] el [elemento] que ubica y orienta la cláusula en su contexto".

En consecuencia de todo lo desarrollado en este apartado, la definición de Tema que se adopta en el presente artículo es la que propone Martin (1992a): Tema es la 
función que orienta a los lectores y oyentes a un ángulo del campo, definición que resuena con la de Halliday y Matthiessen (2014) recién mencionada.

Por otra parte, es necesario abordar el significado y el medio de realización de la otra función textual que Halliday ([1979] 2002) considera relevante: el Nuevo. Aunque pertenece a otro sistema, el de información, el Nuevo interactúa en la cláusula con el Tema para formar lo que Martin y Rose (2007: 188), siguiendo la metáfora de Pike (1982), llaman "pequeñas ondas en el texto".

Antes de avanzar sobre este punto, cabe señalar que los sistemas textuales en el estrato léxico-gramatical son dos: tema e información. El sistema de tema consiste en dos opciones, la de Tema, definida de acuerdo con lo anteriormente discutido, y la de Rema, definida como "el resto del mensaje; la parte en la que el Tema se desenvuelve" (Halliday, 1994: 37).

El sistema de información tiene dos opciones: lo Dado, información ya ofrecida por el texto, "lo que es presentado como conocido por el oyente" (Halliday, 2004: 59) y el Nuevo. En cuanto a su significado, el Nuevo se define como "la información que el oyente/lector es invitado a tomar en cuenta como importante; lo que constituye una novedad", en oposición a lo Dado (Halliday, 1994). Su medio de realización es la entonación (Halliday, 1979 [2002]) y en el caso no marcado, coincide con el último constituyente de la cláusula, que recibe el mayor movimiento tonal (Halliday, 1994). Para simplificar el análisis de Nuevo, Martin (1992b) propone considerar solo el menor dominio de Nuevo, esto es, el último elemento experiencial de la cláusula, sobre el cual recae el mayor pico de prominencia tonal. Esto es especialmente útil para el texto escrito, en el que solo puede considerarse cómo sería leído en voz alta. Estas serán la definición y el medio de realización tomados provisionalmente en este trabajo para Nuevo en español, hasta tanto haya estudios que permitan confirmar o rechazar esta postura.

En términos discursivos, Fries (1981) afirma que la serie de Remas de las cláusulas de un párrafo construyen el punto del texto, que Martin (1992a) considera una fuente de apertura, lo que elabora el campo. Si bien Fries (1981) habla de Rema, tomamos la posición de Halliday ([1979] 2002), que considera que es el Nuevo el complemento del Tema para la construcción de textura. La relación entre ambos es "lo que da periodicidad al discurso" (Halliday ([1979] 2002: 208). Así, en el análisis de los textos consideraremos la interacción entre Tema y Nuevo para la construcción del método de desarrollo y el punto del texto, tal como proponen Martin (1992b) y Martin y Rose (2007). 


\section{Metodología}

En primer lugar, y como ya se señaló antes, es necesario, para comenzar a trabajar, la definición acerca de la metafunción en la que se centrará la investigación, el estrato y el rango. Al estudiar la función de Tema, el foco estará puesto en la metafunción textual y en el estrato léxico-gramatical, específicamente en el rango de la cláusula, a la que la función en juego pertenece.

Sin embargo, al adoptar la perspectiva trinocular de investigación, en la que cada función se aborda desde arriba, desde alrededor y desde abajo (Caffarel et al., 2004a; Matthiessen \& Halliday, 2009; Martin et al., en prensa), se debe tener en cuenta la relación de la función estudiada con la variable de registro correspondiente y con el estrato semántico-discursivo para la mirada desde arriba; las relaciones con otras funciones en el estrato léxico-gramatical en el rango de la cláusula, para la mirada desde alrededor; y la relación con el rango de la palabra para el estudio desde abajo, a fin de considerar la participación de unidades de ese rango en la realización de la función bajo análisis.

Así, para estudiar la función de Tema en español, se tendrán en cuenta los aspectos que se detallan a continuación. Para la mirada desde arriba, se identificarán primero las dimensiones del modo del registro que se verifican en el texto a analizar. Luego, en el estrato semántico-discursivo, se observará el sistema de periodicidad, que da cuenta del flujo de información en el discurso (Martin \& Rose, 2007). En ese sistema, se tendrá en cuenta de qué manera los patrones de Tema en el rango de la cláusula contribuyen a la construcción de patrones de cadenas léxicas o referenciales, es decir, de unidades más abstractas en el discurso, como el método de desarrollo y el punto del texto. Para la mirada desde alrededor, se prestará atención a las relaciones entre Tema y Nuevo para establecer las relaciones entre estas funciones en la cláusula; pero, además, se volverá a la mirada desde arriba para identificar cómo los patrones de Tema se vinculan con los patrones de Nuevo en el discurso, es decir, cómo se relacionan el método de desarrollo y el punto del texto. Finalmente, para la mirada desde abajo, se indagará si algún elemento del rango de la palabra en el estrato léxicogramatical participa en marcar el elemento de la cláusula que realiza la función de Tema.

En lo que hace a la metafunción textual, la cláusula se define como mensaje, lo que significa que se estructura como un 'quantum de información' con ondas de prominencia y ondas de no prominencia (Caffarel et al., 2004). Las ondas de prominencia textual tienen que ver con las funciones de Tema y Nuevo, correspondientes a los sistemas de tema y de información, respectivamente. Existe, sin embargo, una zona de no prominencia, que generalmente incluye al Predicador realizado por el grupo verbal, que puede contribuir al Nuevo cuando éste se extiende más allá del último elemento experiencial de la cláusula, como se define el mínimo- 
Nuevo para trabajar con texto escrito (Martin, 1992b). La cláusula se inicia con la posibilidad de incluir un Tema textual y uno interpersonal, así como con un Tema experiencial marcado, que junto con el Tema no marcado constituyen la zona de prominencia temática. De ahí que los ejemplos se presenten en tablas organizadas cláusula por cláusula en sucesivas líneas y en columnas que identifican estas funciones así como la zona de no prominencia y la unidad que realiza el Nuevo. De esta manera, se intenta graficar la estructura en ondas de la metafunción textual. En casos en que el Tema confla, es decir, coincide en su realización, con el Nuevo (caso que se explicará más abajo), entonces se agrega una columna para indicarlo. La zona de prominencia textual quedará interrumpida por la zona de no prominencia, de modo que el despliegue de la onda se dará de manera diferente a la cláusula en inglés (Moyano, 2016).

En este trabajo se consideran cláusulas analizables para Tema a las independientes, que se identifican en los ejemplos siguiendo el criterio ideacional (Martin, Matthiessen $\&$ Painter, 2010). Este criterio indica que donde hay un Proceso con sus participantes y a veces una Circunstancia, entonces hay una cláusula. En español, el Proceso puede estar elidido, y en esos casos se lo repone a partir del co-texto, esto es, de las cláusulas anteriores. Asimismo, es frecuente la elipsis de participantes, que también se infiere por co-texto y se indica en el análisis mediante el símbolo ' $\varnothing$ ' más la recuperación de su significado entre corchetes simples.

Se entiende por cláusulas independientes las que pueden funcionar por sí solas como una oración. Las cláusulas dependientes no son consideradas para Tema, a excepción de las de gerundio, que proponen una continuidad en relación con las principales y para las cuales se puede identificar el participante central. Cuando una cláusula dependiente precede la independiente, se la considera de manera similar a las Circunstancias, con función de Tema marcado (Martin, 1992a). En estos casos, la cláusula independiente es analizada para Tema, en la consideración de que Tema marcado y Tema no marcado cumplen diferentes funciones en el texto (Martin \& Rose, 2007).

Las cláusulas incrustadas se indican con doble corchete y no son consideradas para el análisis de Tema dado que sufren cambio de rango para funcionar como parte de un componente de la cláusula en la metafunción ideacional. Las cláusulas incluidas, que interrumpen el hilo de la cláusula, son marcadas con el símbolo ' $<<\ldots>>$ ' y no son consideradas para el análisis de Tema. 


\section{Resultados}

\subsection{Medio de realización del Tema en español}

En un capítulo dedicado a la comparación de medios de realización por metafunción entre lenguas, Matthiessen (2004) plantea tres diferentes medios para la metafunción textual: la entonación, la posición y la segmentación. La entonación es fundamentalmente el medio de realización para la función de Nuevo (Halliday, [1979] 2002), a excepción de algunos casos en que el Tema se señala de esta forma. No se tratarán aquí esos casos de excepción. La posición es el medio por el cual se realiza el Tema en inglés, como caso típico. Se trata de que la posición inicial indica la prominencia temática sin otro elemento que lo señale, tal como lo propone Halliday (1994) y como se describe por ejemplo en Caffarel et al. (2004a). La segmentación es el medio de realización mediante el cual el Tema de la cláusula es indicado por una partícula, como ocurre en tagalog (Martin, 2004) o en japonés (Teruya, 2004). Pero ¿cuál de todos estos recursos -si alguno de ellos- resulta el medio de realización del elemento que funciona como Tema de la cláusula en español?

Para responder a esta pregunta es necesario analizar un conjunto de textos con la metodología propuesta por la tipología lingüística en el marco de la LSF, cuyos principios fueron sintetizados en el apartado 3. Los textos son instancias de diferentes géneros, que se irán mencionando a medida que se vayan presentando.

Desde la perspectiva del modo del registro -que representa el primer paso para la mirada desde arriba para analizar la metafunción textual- los textos pueden ser monológicos o dialógicos, según incluyan o no recursos de interacción inmediata entre interlocutores, y pueden representar lenguaje en acción o lenguaje como reflexión según su dependencia contextual (Martin et al., en prensa). En todos los ejemplos que se toman en este trabajo, se trata de textos monológicos y reflexivos -constitutivos de la actividad-, independientes del contexto.

En primer lugar, se toma como ejemplo el fragmento inicial del cuento de Horacio Quiroga, "A la deriva" (Texto 1)3. 
Texto 1. Tema no marcado: cadena léxica que construye el método de desarrollo.

\begin{tabular}{|c|c|c|c|c|c|c|}
\hline & T txt & $\begin{array}{l}\mathrm{T} \\
\text { interp }\end{array}$ & $\begin{array}{l}\mathrm{T} \text { exper } \\
\text { marcado }\end{array}$ & $\begin{array}{l}\text { T exper } \\
\text { no marcado }\end{array}$ & $\begin{array}{l}\text { Zona de no } \\
\text { prominenci } \\
\text { a }\end{array}$ & Nuevo \\
\hline $\mathrm{a}$ & & & & El hombre & pisó & algo blancuzco, \\
\hline $\mathrm{b}$ & $\mathrm{y}$ & & en seguida & $\begin{array}{ll}\varnothing & {[\mathrm{el}} \\
\text { hombre] }\end{array}$ & sintió & la mordedura en el pie. \\
\hline c & & & & $\begin{array}{ll}\varnothing & {[\mathrm{el}} \\
\text { hombre] } & \\
\end{array}$ & Saltó & adelante, \\
\hline d & $\mathrm{y}$ & & $\begin{array}{l}\text { al volverse, } \\
\text { con } \quad \text { un } \\
\text { juramento }\end{array}$ & $\begin{array}{l}\emptyset \\
\text { hombre] }\end{array}$ & vio & una yaracacusú \\
\hline $\mathrm{e}$ & que, & & & $\begin{array}{l}\text { que (la } \\
\text { yacurusú), } \\
\text { [[arrollada } \\
\text { sobre sí } \\
\text { misma]], }\end{array}$ & esperaba & otro ataque. \\
\hline $\int$ & & & & & & \\
\hline $\mathrm{f}$ & & & & El hombre & echó & una veloz ojeada a su pie, \\
\hline g & $\begin{array}{l}\text { dond } \\
e\end{array}$ & & donde & $\begin{array}{l}\text { dos gotitas } \\
\text { de sangre }\end{array}$ & engrosaban & dificultosamente, \\
\hline $\mathrm{h}$ & $\mathrm{y}$ & & & $\begin{array}{ll}\varnothing & {[\mathrm{el}} \\
\text { hombre] } & \end{array}$ & sacó & el machete de la cintura. \\
\hline i & & & & La víbora & vio & la amenaza \\
\hline j & $\mathrm{y}$ & & & $\varnothing$ [la víbora] & hundió & $\begin{array}{l}\text { más la cabeza en el centro } \\
\text { mismo de su espiral; }\end{array}$ \\
\hline $\mathrm{k}$ & pero & & & el machete & cayó & de lomo, \\
\hline 1 & & & & $\begin{array}{l}\varnothing \\
\text { machete] }\end{array}$ & dislocándole & las vértebras. \\
\hline
\end{tabular}

En el Texto 1 se puede observar, desde la perspectiva desde arriba, que la serie de Temas no marcados de ambos párrafos presentan los personajes de la historia como método de desarrollo del texto. Así, tienen como función iluminar el aspecto del campo sobre el cual el autor tiene interés en anclar el texto. En este ejemplo, como en muchos otros casos en español, las cadenas léxicas que construyen el método de desarrollo del texto se ubican al inicio de las cláusulas, pero se observará más adelante que esto no siempre ocurre de la misma manera.

Con perspectiva desde alrededor, se observa que en la función de mínimo-Nuevo en el texto, es decir el último elemento experiencial de la cláusula sobre el que recae el pico tonal más alto, aparece la información sobre la cual se quiere llamar la atención del lector a fin de completar la escena. Si se extiende el Nuevo hacia la izquierda, incluyendo la zona de no prominencia, se completa la información nueva que permite dar un panorama más claro de la escena.

Si se vuelve a la construcción del método de desarrollo en este texto, es importante observar otro fenómeno. En la cláusula (b) se observa que el participante con función de Tema aparece elidido en una cláusula en relación de parataxis con la cláusula (a). 
Este fenómeno es muy frecuente en otras lenguas, como por ejemplo en inglés (Martin et al., en prensa) o en chino (Halliday \& McDonald, 2004). Pero se observa también en las cláusulas (c) y (d), que corresponden a otro complejo clausular. Este fenómeno no se da en inglés, pero en español es absolutamente corriente. Cabe señalar aquí que otros autores (Taboada, 1995; McCabe, 1999; MontemayorBorsinger, 2009; Arús, 2010, 2017; Lavid et al., 2010) no consideran la existencia de un Tema elidido en español sino que entienden que esta función se realiza a través del sufijo verbal, que indica la persona y número del verbo y, por lo tanto, realiza también el Sujeto de la función interpersonal. Esta opción la toman pese a señalar que el Tema en español se realiza por posición, esto es, que el primer elemento experiencial de la cláusula es el que se selecciona como Tema. Esto significa, como lo indican Taboada (1995) y Lavid et al. (2010), que son el Proceso más el sufijo verbal los que realizan la función de Tema en estas cláusulas. Resulta difícil, entonces, considerar el método de desarrollo del texto como una función discursiva orientadora acerca del aspecto del campo que interesa destacar en el texto en cuestión. Gutiérrez (2011), señala en cambio que el Proceso no es candidato a la función de Tema sino solo el sufijo verbal. La interpretación que aquí se sostiene implica la existencia de Temas explícitos y Temas elididos o implícitos, que permiten construir el método de desarrollo del texto (cláusulas (h) y (j)). El caso de la cláusula final del fragmento es importante pues representa un caso diferente: hay cláusulas de gerundio que permiten continuar el desarrollo del texto y que permiten identificar el participante que se selecciona como Tema (Moyano, 2010, 2016; Martin et al., en prensa).

Como segundo ejemplo, se presenta un breve texto del campo de la historia (Texto 2), tomado de un manual de educación primaria (Ciencias Sociales 5, 2004). El género es una explicación histórica, que presenta eventos relacionados temporal y causalmente, con énfasis en las relaciones causales. 
Texto 2. Temas explícitos e implícitos que construyen el método de desarrollo.

\begin{tabular}{|c|c|c|c|c|c|c|}
\hline & T txt & $\begin{array}{l}\mathbf{T} \\
\text { interp }\end{array}$ & $\begin{array}{l}\mathrm{T} \text { exper } \\
\text { marcado }\end{array}$ & $\begin{array}{l}\text { T exper } \\
\text { no marcado }\end{array}$ & $\begin{array}{l}\text { Zona de no } \\
\text { prominencia }\end{array}$ & Nuevo \\
\hline $\mathrm{a}$ & & & $\begin{array}{l}\text { Mientras } \\
\text { tanto, }\end{array}$ & $\begin{array}{ll}\text { todos } & \text { los } \\
\text { unitarios } & \end{array}$ & $\begin{array}{l}\text { tenían puestas } \\
\text { sus esperanzas }\end{array}$ & en el general Paz. \\
\hline b & & & $\begin{array}{l}\text { Desde } \\
\text { Córdoba, }\end{array}$ & Paz & $\begin{array}{l}\text { había derrotado a } \\
\text { Quiroga }\end{array}$ & $\begin{array}{l}\text { en La Tablada y en } \\
\text { Oncativo, }\end{array}$ \\
\hline c & $\mathrm{y}$ & & & $\varnothing[\mathrm{Paz}]$ & $\begin{array}{l}\text { estableció } \\
\text { gobiernos } \\
\text { unitarios }\end{array}$ & $\begin{array}{ll}\text { en todas las } \\
\text { provincias } & \text { del } \\
\text { interior. } & \end{array}$ \\
\hline \multicolumn{7}{|l|}{$\int$} \\
\hline d & & & $\begin{array}{l}\text { El 31 de } \\
\text { agosto de } \\
\underline{1830,}\end{array}$ & los unitarios & conformaron & la Liga del Interior, \\
\hline $\mathrm{e}$ & $\mathrm{y}$ & & & $\varnothing[$ los unitarios $]$ & se dispusieron & $\begin{array}{l}\text { a [[derrotar a las } \\
\text { provincias } \\
\text { federales]]. }\end{array}$ \\
\hline \multicolumn{7}{|l|}{ S } \\
\hline $\mathrm{f}$ & & & $\begin{array}{l}\text { Como } \\
\text { respuesta }\end{array}$ & $\begin{array}{lr}\text { Buenos } & \text { Aires, } \\
\text { Santa Fe y } & \text { Entre } \\
\text { Ríos } & << \\
\text { (Corrientes } & \text { se } \\
\text { incorporó } & \text { más } \\
\text { tarde) }>> & \\
\end{array}$ & firmaron & $\begin{array}{l}\text { el Pacto Federal en } \\
\text { enero de } 1831 .\end{array}$ \\
\hline $\mathrm{g}$ & & & & El Pacto & estableció & $\begin{array}{l}\text { [[que las provincias } \\
\text { firmantes se unían } \\
\text { en una alianza ante } \\
\text { el ataque de } \\
\text { cualquier } \\
\text { provincia]]. }\end{array}$ \\
\hline \multicolumn{7}{|l|}{ 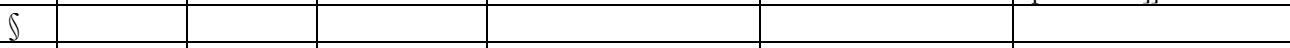 } \\
\hline $\mathrm{h}$ & $\begin{array}{l}\text { Tam- } \\
\text { bién }\end{array}$ & & & $\varnothing$ [E1 Pacto] & se ocupó & $\begin{array}{lr}\text { de la organización } \\
\text { constitucional del } \\
\text { país. }\end{array}$ \\
\hline i & & & & $\varnothing$ [E1 Pacto] & estableció & $\begin{array}{l}\text { la reunión de una } \\
\text { Comisión } \\
\text { representativa } \\
\text { Santa Fe }\end{array}$ \\
\hline$j$ & que & & & $\begin{array}{l}\text { que (una } \\
\text { Comisión } \\
\text { representativa en } \\
\text { Santa Fe) }\end{array}$ & debía convocar & $\begin{array}{l}\text { a un Congreso [[que } \\
\text { organizara el país } \\
\text { bajo el sistema } \\
\text { federal]]. }\end{array}$ \\
\hline
\end{tabular}

En el Texto 2 se observa con claridad de qué manera la serie de Temas no marcados de las diferentes cláusulas se identifican desde arriba porque construyen el método de desarrollo del texto. En este caso, el aspecto del campo que se destaca son las dos facciones políticas que en el período de organización nacional -desde los años 20 hasta los 60 del siglo XIX- en las Provincias Unidas del Río de la Plata (lo que después fue la República Argentina) se oponían entre sí: los llamados 'unitarios', y los llamados 'federales'. De hecho, se seleccionan como Tema participantes como 'todos 
los unitarios', 'los unitarios' y 'Paz', uno de los representantes más relevantes de ese sector, en el primer párrafo, mientras que el segundo y el tercero seleccionan las provincias más importantes entre las federales así como 'el Pacto', documento fundacional de la alianza federal y, finalmente, un pronombre que refiere a 'una Comisión representativa [de los federales] en Santa Fe'. Obsérvese que también aparecen Temas elididos en las cláusulas (c) y (e), en relación paratáctica con cada una de las anteriores. Sin embargo, a diferencia del inglés, en español es posible que el Tema elidido aparezca no solo en complejos clausulares diferentes sino incluso en una cláusula que inicia un párrafo. Como en chino, la recuperación del significado del participante que funciona como Tema se hace por co-texto.

En cuanto a la mirada desde alrededor, puede afirmarse lo mismo que con respecto al Texto 1: la serie de Nuevos de las diferentes cláusulas va construyendo el punto del texto, consistente esta vez en los avances de cada una de las facciones políticas en juego en las Provincias Unidas del Río de la Plata de aquella época.

El análisis de ambos textos demanda una observación acerca de la extensión del Tema. Se considera en esta descripción que el Tema marcado y el Tema no marcado son considerados como zona temática en los textos en español, de la misma manera que Martin y Rose (2007) los consideran para el inglés, dado que cumplen dos funciones diferentes pero esenciales desde la perspectiva textual: el Tema no marcado andamia la continuidad en el discurso, mientras el Tema marcado andamia la discontinuidad. El Tema marcado, que en español, como se verá más adelante, se realiza mediante una Circunstancia en los casos más frecuentes, cumple la función de puntuar el texto en términos de tiempo y lugar, fundamentalmente, mientras el Tema no marcado la de mantener el método de desarrollo del texto. Pero sobre este aspecto se volverá más adelante para abordarlo en detalle.

En cuanto al Tema implícito, es interesante analizar el Texto 3, un fragmento del cuento de Borges 'Los dos reyes y los dos laberintos'. 
Texto 3. Uso del Tema implícito en español.

\begin{tabular}{|c|c|c|c|c|c|c|c|}
\hline & T txt & T interp & $\begin{array}{l}\mathrm{T} \text { exper } \\
\text { marcado }\end{array}$ & \begin{tabular}{|l|} 
T exper \\
no \\
marcado
\end{tabular} & $\begin{array}{l}\text { Zona de no } \\
\text { prominencia }\end{array}$ & Tema/Nuevo & Nuevo \\
\hline $\mathrm{a}$ & & & $\begin{array}{l}\text { Con el } \\
\text { andar del } \\
\text { tiempo }\end{array}$ & & vino a su corte & $\begin{array}{l}\text { un rey de los } \\
\text { árabes, }\end{array}$ & \\
\hline $\mathrm{b}$ & $\mathrm{y}$ & & & $\begin{array}{l}\text { el rey de } \\
\text { Babilonia }\end{array}$ & $\begin{array}{llr}<<\text { (para hacer } \\
\text { burla de la } \\
\text { simplicidad de } & \text { su } \\
\text { huésped) }>> & \text { lo } \\
\text { hizo penetrar } & \end{array}$ & & en el laberinto \\
\hline c & donde & & donde & $\begin{array}{lll}\text { O } & \text { [un } & \text { rey } \\
\text { de } & \text { los } \\
\text { árabes] }\end{array}$ & $\begin{array}{l}\text { vagó afrentado y } \\
\text { confundido }\end{array}$ & & $\begin{array}{ll}\text { hasta } & \text { la } \\
\text { declinación } & \text { de } \\
\text { la tarde. } & \\
\end{array}$ \\
\hline d & $\begin{array}{l}\text { Enton- } \\
\text { ces }\end{array}$ & & & 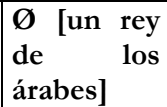 & imploró & & socorro divino \\
\hline $\mathrm{e}$ & $\mathrm{y}$ & & & \begin{tabular}{|lrr}
$\varnothing$ & [un & rey \\
de & los \\
árabes] & \\
\end{tabular} & dio & & con la puerta. \\
\hline$\sqrt{S}$ & & & & Sus labios & no profirieron & & queja ninguna, \\
\hline $\mathrm{f}$ & pero & & & $\begin{array}{lll}\emptyset & \text { [un } & \text { rey } \\
\text { de } & \text { los } \\
\text { árabes] } & \end{array}$ & le dijo & & $\begin{array}{lcc}\text { al rey } & \text { de } \\
\text { Babilonia } & \end{array}$ \\
\hline g & que & & & él & en Arabia tenía & & otro laberinto \\
\hline $\mathrm{h}$ & y que, & $\begin{array}{l}\text { si Dios } \\
\text { era } \\
\text { servido, }\end{array}$ & & [él] & se lo daría a conocer & & algún día. \\
\hline i & & & Luego & 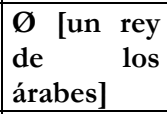 & regresó & & a Arabia, \\
\hline j & & & & \begin{tabular}{|lrr}
$\varnothing$ & [un & rey \\
de & los \\
árabes] & \\
\end{tabular} & juntó & & $\begin{array}{l}\text { sus capitanes y } \\
\text { sus alcaides }\end{array}$ \\
\hline $\mathrm{k}$ & $\mathrm{y}$ & & & 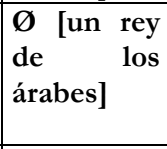 & estragó & & $\begin{array}{l}\text { los reinos de } \\
\text { Babilonia con } \\
\text { tan venturosa } \\
\text { fortuna }\end{array}$ \\
\hline 1 & que & & & \begin{tabular}{|lrr} 
& [un & rey \\
de & los \\
árabes] & \\
\end{tabular} & derribó & & sus castillos, \\
\hline $\mathrm{m}$ & & & & $\begin{array}{lll}\varnothing & \text { [un } & \text { rey } \\
\text { de } & \text { los } \\
\text { árabes] } & \\
\end{array}$ & rompió & & sus gentes \\
\hline $\mathrm{n}$ & e & & & $\begin{array}{lll}\varnothing & \text { [un } & \text { rey } \\
\text { de } & \text { los } \\
\text { árabes] } & \end{array}$ & hizo cautivo & & al mismo rey. \\
\hline $\mathrm{o}$ & & & & $\begin{array}{lll}\varnothing & \text { [un } & \text { rey } \\
\text { de } & \text { los } \\
\text { árabes] } & \end{array}$ & Lo amarró & & $\begin{array}{l}\text { encima de un } \\
\text { camello veloz }\end{array}$ \\
\hline p & & & & 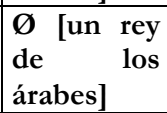 & y lo llevó & & al desierto. \\
\hline
\end{tabular}


En este texto es notable el uso de Temas implícitos, que se recuperan por co-texto, junto con el significado del pronombre 'él' en la cláusula (g), del último elemento experiencial de la cláusula (a): 'un rey de los árabes'. Desde una perspectiva desde arriba, esto permite decir que el Nuevo de la cláusula (a) inaugura el método de desarrollo del texto, constituido mayormente -a excepción del Tema de la cláusula (b)por un pronombre y elipsis que recuperan su significado. Esto permite decir, entonces, que el elemento que realiza el Nuevo en la cláusula no solo contribuye al punto sino al método de desarrollo del texto, lo que lo colocaría en función de Tema no marcado, conflando con el Nuevo.

El análisis de estos primeros tres textos, entonces, habilita a afirmar, desde arriba, que el Tema en español se realiza mediante un participante que contribuye al método de desarrollo del texto, ya sea que esté explícito o elidido -y entonces implícito-, y si está explícito, que puede preceder o estar postpuesto al Predicador (para la función interpersonal de Predicador, Quiroz, 2015).

Véase, para abundar sobre esta afirmación, el Texto 4, tomado de una enciclopedia (Cousteau, 1994), que instancia un informe procedimental genérico (Moyano, 2013). 
Texto 4. Tema no marcado conflado con Nuevo.

\begin{tabular}{|c|c|c|c|c|c|c|c|}
\hline & \begin{tabular}{|l|}
$T$ \\
txt
\end{tabular} & \begin{tabular}{|l|}
$\mathrm{T}$ \\
interp
\end{tabular} & $\begin{array}{l}T \text { exper } \\
\text { marcado }\end{array}$ & \begin{tabular}{|l} 
T exper \\
no \\
marcado \\
\end{tabular} & $\begin{array}{l}\text { Zona de no } \\
\text { prominenci } \\
\text { a }\end{array}$ & Tema/Nuevo & Nuevo \\
\hline $\mathrm{a}$ & & & $\begin{array}{l}\text { Una vez en el } \\
\text { laboratorio de } \\
\text { a bordo, }\end{array}$ & $\begin{array}{|lr|}\text { el } & \text { testigo } \\
\text { [[obtenido } \\
\text { en r el } \\
\text { fondo del } \\
\text { mar]] }\end{array}$ & $\begin{array}{l}\text { es sacado de } \\
\text { su tubo }\end{array}$ & & $\begin{array}{l}\text { por medio de } \\
\text { un pistón, }\end{array}$ \\
\hline $\mathrm{b}$ & $\mathrm{O}$ & & & & & & $\begin{array}{ll}\text { abriendo } & \text { la } \\
\text { funda } & \text { de } \\
\text { plástico. } & \\
\end{array}$ \\
\hline $\mathrm{c}$ & $\mathrm{Y}$ & & & $\begin{array}{l}\emptyset \\
\text { testigo] }\end{array}$ & $\begin{array}{l}\text { se parte en } \\
\text { dos }\end{array}$ & & $\begin{array}{l}\text { longitudinalme } \\
\text { nte: }\end{array}$ \\
\hline $\mathrm{d}$ & & & & $\begin{array}{l}\text { una de las } \\
\text { dos } \\
\text { mitades }\end{array}$ & $\begin{array}{l}\text { se guarda } \\
\text { inmediatame } \\
\text { nte }\end{array}$ & & $\begin{array}{l}\text { en un } \\
\text { receptáculo } \\
\text { [[etiquetado]], }\end{array}$ \\
\hline $\mathrm{e}$ & $y$ & & & $\begin{array}{l}\emptyset \quad[\text { esa } \\
\text { mitad] }\end{array}$ & $\begin{array}{l}\text { se conserva a } \\
4^{\circ} \mathrm{C}\end{array}$ & & $\begin{array}{l}\text { como muestra } \\
\text { de referencia. }\end{array}$ \\
\hline$f$ & & & & $\begin{array}{l}\text { La otra } \\
\text { mitad }\end{array}$ & & & es fotografiada \\
\hline $\mathrm{g}$ & $y$ & & & $\begin{array}{l}\emptyset[\mathrm{La} \text { otra } \\
\text { mitad] }\end{array}$ & & & [es] descrita; \\
\hline $\mathrm{h}$ & & & & & se anotan & sus colores & $\begin{array}{l}\text { después de } \\
\text { [[compararlos } \\
\text { con los de una } \\
\text { escala } \\
\text { cromática } \\
\text { calibrada]]. } \\
\end{array}$ \\
\hline i & & & & & $\begin{array}{l}\text { Se examinan } \\
\text { con } \\
\text { particular } \\
\text { atención }\end{array}$ & $\begin{array}{l}\text { la sucesión } \\
\text { característica de los } \\
\text { estratos, la estructura de } \\
\text { la muestra, la presencia } \\
\text { de restos de animales o } \\
\text { de plantas (conchas, } \\
\text { caparazones...), la } \\
\text { forma y dimensión de } \\
\text { las partículas rocosas, } \\
\text { etc. }\end{array}$ & \\
\hline j & & & $\begin{array}{l}\text { Si } \text { esta labor } \\
\text { es llevada a } \\
\text { cabo con } \\
\text { minuciosidad } \\
\text { y método, } / /\end{array}$ & & $\begin{array}{l}\text { se pueden } \\
\text { reconstruir } \\
\text { con la } \\
\text { imaginación }\end{array}$ & $\begin{array}{l}\text { las condiciones en que } \\
\text { se formó la muestra: } \\
\text { temperatura, presión, } \\
\text { humedad, salinidad, } \\
\text { tipo de atmósfera. }\end{array}$ & \\
\hline $\mathrm{k}$ & & & \begin{tabular}{|l} 
Por medio de \\
radiografías \\
\end{tabular} & & se precisan & $\begin{array}{ll}\text { las } & \text { estructuras } \\
\text { constitutivas del testigo }\end{array}$ & \\
\hline 1 & $\mathrm{y}$ & & & & se descubren & $\begin{array}{l}\text { otras, invisibles a simple } \\
\text { vista. }\end{array}$ & \\
\hline $\mathrm{m}$ & & & $\begin{array}{l}\text { Sacando } \\
\text { laminillas de } \\
\text { la muestra // } \\
\text { la } \\
\text { y } \\
\text { observándolas } \\
\begin{array}{ll}\text { a la luz } \\
\text { polarizada,// }\end{array}\end{array}$ & & $\begin{array}{l}\text { se pueden } \\
\text { poner de } \\
\text { manifiesto }\end{array}$ & $\begin{array}{l}\text { las formas y disposición } \\
\text { de partículas nuevas }\end{array}$ & \\
\hline
\end{tabular}


En el Texto 4, siguiendo la línea de análisis desde arriba que se viene haciendo en este trabajo, se pueden observar dos momentos en el texto: uno en el que el método de desarrollo del texto se construye mediante Temas que refieren al testigo o muestra y a sus dos mitades; otro en el que el Tema confla con el Nuevo y refiere a lo que se observa o se construye mediante observación de la parte del testigo que se analiza. Esto tiene sentido en el texto, ya que a continuación -aunque no hay espacio para mostrarlo aquí- se construye una justificación que refiere al valor de este tipo de investigaciones por las conclusiones a las que permite arribar a partir de las observaciones realizadas. Así, el punto de la primera parte construye lo que se 'hace' con la mitad del testigo que se analiza, mientras la segunda parte del punto refiere a lo que se puede observar y concluir a partir de ese análisis.

Por otro lado, en este texto, los Temas no marcados puntúan el texto en términos de tiempo (cláusula (a)), condición (cláusula (j)) y medio o modo (cláusulas (k) y (m)), marcando discontinuidad.

En este punto, se planteará una mirada desde abajo, es decir, teniendo en cuenta otros rangos del estrato léxico-gramatical en el análisis. Si la realización de Tema en español admite tantas posibilidades cuando se la mira desde arriba, ¿cuál es su medio de realización?, ¿cómo se gramaticaliza el Tema en español?

Evidentemente, volviendo a la síntesis de Matthiessen (2004), el Tema en español no se realiza por posición puesto que puede anteceder o estar postpuesto al Predicador. Tampoco es secuencial, pues no hay ninguna partícula que indique el elemento que funciona como Tema, de la manera que ocurre en tagalog (Martin, 2004) o en japonés (Teruya, 2004). La única respuesta posible para el español, es que tiene un medio de realización diferente, que es el de concordancia. Si se observan los textos del 1 al 4, lo que muestran en común los elementos que funcionan como Tema en esta lengua es que son participantes que concuerdan con el sufijo verbal en número y persona.

Se propone, entonces, en este trabajo, que el medio de realización del Tema en español es la concordancia. El Tema está realizado por un participante, explícito o implícito, antepuesto o postpuesto al Predicador, que concuerda con el sufijo verbal, ubicado en el rango de la palabra. La realización del Tema por un participante contribuye a la generalización propuesta por Rose (2001).

El sufijo verbal en español, entonces, cumple la función textual de ser indicador del Tema en español. No realiza el Tema sino que lo marca. La función de Tema está realizada por el participante seleccionado por el sufijo verbal, que en la metafunción ideacional cumple la función de ser el Participante central en la cláusula, ya sea en voz activa o pasiva y que no cumple función en la metafunción interpersonal, siguiendo a Quiroz (2008, 2013, 2015). De esta manera, se agrega un cuarto medio de realización 
de la metafunción textual a los propuestos por Matthiessen (2004). Este medio es la concordancia en número y persona entre el grupo nominal y el sufijo verbal.

A continuación, es necesario abordar una cuestión que ha sido tratada por autores como Taboada (1995), Montemayor-Borsinger (2009) y Arús (2017). Se trata de la consideración de los clíticos como Tema. La posición que sostiene este artículo rechaza esa posibilidad, según se mostrará en el Texto 5, tomado del cuento "El brujo postergado", de Borges.

Texto 5. Los clíticos como parte de la zona de no prominencia textual.

\begin{tabular}{|c|c|c|c|c|c|c|c|}
\hline & T txt & $\begin{array}{l}\mathrm{T} \\
\text { interp }\end{array}$ & $\begin{array}{l}\mathrm{T} \text { exper } \\
\text { marcado }\end{array}$ & $\begin{array}{l}\text { T exper } \\
\text { no marcado }\end{array}$ & $\begin{array}{l}\text { Zona de no } \\
\text { prominencia }\end{array}$ & $\begin{array}{l}\text { Tema/ } \\
\text { Nuevo }\end{array}$ & Nuevo \\
\hline $\mathrm{a}$ & & & $\begin{array}{l}\text { En } \\
\text { Santiago }\end{array}$ & & había & & $\begin{array}{l}\text { un deán [[que } \\
\text { tenía codicia de } \\
\text { aprender el arte } \\
\text { de la magia]] }\end{array}$ \\
\hline $\mathrm{b}$ & & & & $\varnothing$ [El deán] & Oyó decir & & \\
\hline c & que & & & $\begin{array}{l}\text { don Illán de } \\
\text { Toledo }\end{array}$ & la sabía & & $\begin{array}{l}\text { más que } \\
\text { ninguno, }\end{array}$ \\
\hline $\mathrm{d}$ & $\mathrm{y}$ & & & Ø [el deán] & fue a Toledo & & a [[buscarlo]]. \\
\hline $\int$ & & & & & & & \\
\hline $\mathrm{e}$ & & & $\begin{array}{l}\text { El día } \\
\text { [[que } \\
\text { llegó]] }\end{array}$ & Ø [el deán] & enderezó & & $\begin{array}{l}\text { a la casa de don } \\
\text { Illán }\end{array}$ \\
\hline $\mathrm{f}$ & $\mathrm{y}$ & & & $\varnothing$ [el deán] & lo encontró & & \\
\hline $\mathrm{g}$ & & & & $\begin{array}{l}\text { Ø [don Illán } \\
\text { de Toledo] }\end{array}$ & leyendo & & $\begin{array}{ll}\text { en una } & \text { ubitación } \\
\text { hapartada. } & \\
\text { apa }\end{array}$ \\
\hline $\int$ & & & & & & & \\
\hline $\mathrm{h}$ & & & & Éste & lo recibió & & con bondad \\
\hline $\mathrm{i}$ & $\mathrm{y}$ & & & $\varnothing$ [éste] & $\underline{\text { le dijo }}$ & & \\
\hline j & que & & & Ø [el deán] & $\begin{array}{ll}\text { postergara } & \text { el } \\
\text { motivo de su } \\
\text { visita }\end{array}$ & & $\begin{array}{l}\text { hasta después } \\
\text { de comer. }\end{array}$ \\
\hline $\mathrm{k}$ & & & & $\begin{array}{l}\emptyset \text { [don Illán } \\
\text { de Toledo] }\end{array}$ & Le señaló & & $\begin{array}{l}\text { un alojamiento } \\
\text { muy fresco }\end{array}$ \\
\hline 1 & $\mathrm{y}$ & & & $\begin{array}{l}\varnothing \text { [don Illán } \\
\text { de Toledo] }\end{array}$ & $\underline{\text { le dijo }}$ & & \\
\hline $\mathrm{m}$ & que & & & & $\underline{\text { lo }}$ mucho alegraba & $\begin{array}{l}\text { su } \\
\text { venida. }\end{array}$ & \\
\hline $\mathrm{n}$ & & & $\begin{array}{l}\text { Después } \\
\text { de comer }\end{array}$ & el deán & le refirió & & $\begin{array}{l}\text { la razón de } \\
\text { aquella visita }\end{array}$ \\
\hline $\mathrm{O}$ & $\mathrm{y}$ & & & $\varnothing$ [el deán] & le rogó & & \\
\hline $\mathrm{p}$ & que & & & $\begin{array}{l}\text { Ø [don Illán } \\
\text { de Toledo] }\end{array}$ & le enseñara & & $\begin{array}{l}\text { la ciencia } \\
\text { mágica. }\end{array}$ \\
\hline
\end{tabular}

Como puede observarse en el análisis, la secuencia de Temas no marcados en el texto se distribuye entre 'el deán’ y 'don Illán de Toledo', los personajes del relato, de manera que construyen el método de desarrollo del texto. Estos participantes están explícitos en algunos casos y elididos en otros, a fin de sostener la cohesión del texto. 
En las cláusulas (h) e (i), es el pronombre 'este' el que funciona como Tema, una vez explícito y otra vez, elidido. En un solo caso, en la cláusula (m), el Tema se encuentra postpuesto, conflando con la función de Nuevo. En muchas de las cláusulas del texto, se marca mediante subrayado la presencia de un clítico en la zona de no prominencia textual. Siguiendo a Quiroz (2013, 2015, 2017), se considera el clítico como parte del grupo verbal que realiza el Predicador, de manera que no funciona en el rango de la cláusula, razón por la cual no puede considerarse candidato para funcionar como Tema. Por otro lado, ya se ha establecido que el Tema no marcado en español se realiza mediante concordancia, es decir, por un participante que funciona en el rango de la cláusula y concuerda con el sufijo verbal en número y persona.

Un tema que merece mayor ejemplificación que hasta el momento es el Tema marcado en español. Como se anticipó, el Tema marcado es, con mucha frecuencia, un Adjunto Circunstancial, que puntúa el texto en tiempo, lugar, condición o medio. En los casos en que aparece un Tema marcado, la zona temática se extiende hasta incluir el Tema no marcado, como ya se explicó antes. Véase el Texto 6 como ejemplo, tomado de la sección Materiales y Métodos de un artículo científico en el campo de las Ciencias Agronómicas. El texto es un informe procedimental (Moyano, 2013). 
Texto 6. Temas marcados andamiando particularmente la discontinuidad temporal en el texto.

\begin{tabular}{|c|c|c|c|c|c|c|c|}
\hline & $\begin{array}{l}\mathrm{T} \\
\mathrm{txt}\end{array}$ & $\begin{array}{l}\text { T } \\
\text { interp }\end{array}$ & $\mathbf{T}$ exper marcado & $\begin{array}{l}\mathrm{T} \text { exper } \\
\text { no marcado }\end{array}$ & $\begin{array}{l}\text { Zona de no } \\
\text { prominen- } \\
\text { cia }\end{array}$ & $\begin{array}{l}\text { Tema/ } \\
\text { Nuevo }\end{array}$ & Nuevo \\
\hline a & & & $\begin{array}{l}\text { Al final del primer } \\
\text { ciclo de } \\
\text { producción (junio) }\end{array}$ & las pasturas & se defoliaron & & en una fecha común \\
\hline $\mathrm{b}$ & & & & & observando & & $\begin{array}{l}\text { las alturas corres- } \\
\text { pondientes a cada } \\
\text { tratamiento. }\end{array}$ \\
\hline & {$[[\cdots]$} & & & & & & \\
\hline$S$ & & & $\begin{array}{ll}\text { En } & \text { cada } \\
\text { defoliación, } & \end{array}$ & $\begin{array}{l}\text { la } \\
\text { acumulación } \\
\text { de forraje }\end{array}$ & fue estimada & & $\begin{array}{l}\text { mediante la pesada a } \\
\text { campo del forraje } \\
\text { cortado; }\end{array}$ \\
\hline $\mathrm{d}$ & & & del mismo & & se extrajo & $\begin{array}{l}\text { una muestra } \\
\text { representa-tiva de } \\
0,25 \mathrm{~kg} \text {, }\end{array}$ & \\
\hline $\mathrm{e}$ & que & & & $\begin{array}{l}\text { que (la } \\
\text { muestra) }\end{array}$ & fue secada & & $\begin{array}{l}\text { por } 48 \text { hs en estufa } \\
\text { a } 60^{*} \mathrm{C}\end{array}$ \\
\hline $\mathrm{f}$ & para & & & & Determinar & & $\begin{array}{l}\text { el porcentaje de } \\
\text { materia seca (MS). }\end{array}$ \\
\hline $\int$ & & & & & & & \\
\hline $\mathrm{g}$ & & & $\begin{array}{l}\text { Después de cada } \\
\text { defoliación, }\end{array}$ & & $\begin{array}{l}\text { fueron } \\
\text { ubicados }\end{array}$ & $\begin{array}{l}\text { dos marcos de } 0,06 \\
\text { m2 en cada unidad } \\
\text { experimental, }\end{array}$ & $\begin{array}{l}\text { en la parte central } \\
\text { de los surcos } \\
\text { correspon-dientes a } \\
\text { las mediciones de } \\
\text { variables } \\
\text { estructurales, }\end{array}$ \\
\hline $\mathrm{h}$ & & & & & evitando & & $\begin{array}{l}\text { [[colocarlos en } \\
\text { sectores } \\
\text { previamente } \\
\text { muestrea-dos]]. }\end{array}$ \\
\hline $\mathrm{i}$ & & & En estos marcos, & el forraje & se cortó & & a ras del suelo \\
\hline $\mathrm{j}$ & $\mathrm{y}$ & & en laboratorio & & se determinó & $\begin{array}{l}\text { el número de tallos } \\
\text { (densidad de tallos) }\end{array}$ & \\
\hline & {$[\ldots]$} & & & & & & \\
\hline$S$ & & & $\begin{array}{lr}\text { Al finalizar } & \text { cada } \\
\text { ciclo } & \text { de } \\
\text { producción } & \\
(26 / 6 / 00 & \text { y } \\
25 / 6 / 01) & \\
\end{array}$ & & se examinó & el sistema radical. & \\
\hline j & & & & $\begin{array}{l}\text { Otra } \\
\text { medición } \\
\text { adicional }\end{array}$ & $\begin{array}{l}\text { se efectuó... } \\
{[\ldots]}\end{array}$ & & \\
\hline $\mathrm{k}$ & & & $\begin{array}{l}\text { En } \\
\text { oportunidad, }\end{array}$ & & se extrajo & $\begin{array}{l}\text { un bloque de 0,016 } \\
\text { m3 de suelo sobre un } \\
\text { surco de trébol rojo } \\
\text { con un aparato } \\
\text { diseñado a tal efecto. }\end{array}$ & \\
\hline 1 & & & & $\begin{array}{l}\text { La } \\
\text { profundidad } \\
\text { de muestreo }\end{array}$ & fue & & de $0,2 \mathrm{~m}$. \\
\hline $\mathrm{m}$ & & & $\begin{array}{l}\text { Después } \\
\text { muestreo, }\end{array}$ & el pozo & fue llenado & & $\begin{array}{l}\text { con suelo de } \\
\text { similares caracterís- } \\
\text { ticas al extraído. }\end{array}$ \\
\hline $\mathrm{n}$ & & & & Las raíces & $\begin{array}{l}\text { fueron } \\
\text { lavadas }\end{array}$ & & $\begin{array}{l}\text { cuidadosa-mente } \ldots \\
{[\ldots] .}\end{array}$ \\
\hline $\int$ & & & & & & & \\
\hline$\tilde{\mathrm{n}}$ & & & $\begin{array}{l}\text { En dos } \\
\text { oportunidades, a } \\
\text { los 15 y 39 meses } \\
\text { desde la siembra, }\end{array}$ & & se evaluó & la cobertura del suelo & $\begin{array}{lr}\text { mediante } & \text { el } \\
\text { recuento de los } \\
\text { espacios vacíos }(15 \\
\text { cm lineales) en tres } \\
\text { surcos centrales. }\end{array}$ \\
\hline $\mathrm{o}$ & & & & Los datos & $\begin{array}{l}\text { se expresa- } \\
\text { ron }\end{array}$ & & $\begin{array}{l}\text { como porcentaje de } \\
\text { cobertura de trébol } \\
\text { rojo. }\end{array}$ \\
\hline
\end{tabular}


En el Texto 6 se observa una serie de Temas marcados que puntúan las diferentes acciones llevadas a cabo en el tiempo durante el método aplicado para la construcción de datos en la investigación, con algunos otros que construyen espacio de manera más acotada. Con respecto a los que marcan tiempo, es importante destacar que marcan las etapas de la investigación. Los Temas no marcados, en cambio, construyen como método de desarrollo del texto los objetos que serán estudiados y los datos que se construyen en la investigación. La serie de Nuevos de las cláusulas del texto construyen como punto los detalles de las mediciones que se llevan a cabo en el proceso.

El Texto 6, en consecuencia, permite destacar la función del Tema marcado en español, que tal como lo señalado por Martin y Rose (2007) en inglés es el de andamiar la discontinuidad. Moyano (2010) ya observa este fenómeno y denomina a estos Temas usando la metáfora 'bisagra', en el sentido de dispositivo por el que se logra el cambio.

El próximo texto a observar (Texto 7) es un fragmento del cuento 'Casa tomada', de Julio Cortázar. En él se puede notar el uso de Temas marcados realizados mediante participantes no concordantes, que funcionan cambiando la orientación del texto de manera local, de modo que inciden también en el cambio del método de desarrollo. El fragmento inicia con los personajes y su relación con el dinero y luego va cambiando hacia las actividades que cada uno de los personajes lleva a cabo durante el día.

Texto 7. Participantes no concordantes como Temas marcados.

\begin{tabular}{|c|c|c|c|c|c|c|c|}
\hline & T txt & $T$ interp & $\begin{array}{l}\text { T exper } \\
\text { marcado }\end{array}$ & $\begin{array}{l}\text { T exper } \\
\text { no } \\
\text { marcado }\end{array}$ & & $\begin{array}{l}\text { Tema/ } \\
\text { Nuevo }\end{array}$ & Nuevo \\
\hline $\mathrm{a}$ & & & & $\begin{array}{l}\emptyset \\
\text { [Nosotros] }\end{array}$ & $\begin{array}{l}\text { No } \\
\text { necesitábamos }\end{array}$ & & $\begin{array}{l}\text { [[ganarnos la } \\
\text { vida]], }\end{array}$ \\
\hline $\mathrm{b}$ & & & $\begin{array}{ll}\text { todos } & \text { los } \\
\text { meses } & \\
\end{array}$ & & llegaba & plata & $\begin{array}{ll}\text { de } & \text { los } \\
\text { campos } & \\
\end{array}$ \\
\hline c & $\mathrm{y}$ & & & el dinero & aumentaba. & & \\
\hline $\mathrm{d}$ & Pero & & a Irene & & $\begin{array}{ll}\begin{array}{l}\text { solamente } \\
\text { entretenía }\end{array} & \text { la } \\
\end{array}$ & el tejido, & \\
\hline $\mathrm{e}$ & & & & Ø [Ella] & mostraba & & $\begin{array}{l}\text { una destreza } \\
\text { maravillosa }\end{array}$ \\
\hline $\mathrm{f}$ & $y$ & & a mí & & se me iban & las horas & \\
\hline $\mathrm{g}$ & & & & $\varnothing$ [уо] & $\begin{array}{l}\text { viéndole las } \\
\text { manos }\end{array}$ & & $\begin{array}{l}\text { como erizos } \\
\text { plateados }\end{array}$ \\
\hline
\end{tabular}

El Texto 8, otro fragmento de 'Casa tomada', permite mostrar todos los aspectos ya señalados antes en el análisis textual y confirman, desde arriba, desde alrededor y desde abajo, que el Tema en español es realizado por concordancia. El sufijo verbal selecciona a través de la concordancia en número y persona un participante que, en conjunto con los otros Temas de las cláusulas del texto, construyen el método de 
desarrollo. En este caso, son nuevamente los personajes del relato, incluyendo la misma casa. Asimismo, se observan dos Temas marcados: un Adjunto, que puntúa el texto en términos de tiempo (cláusula (a)) y un participante no concordante, que lo hace de manera local, para cambiar la mirada de un participante a otro (cláusula (d)). La cláusula (l) forma parte del conjunto que no tienen Tema en español (Martin et al., en prensa).

Texto 8. Realización de Temas no marcados y Temas marcados en español.

\begin{tabular}{|c|c|c|c|c|c|c|c|}
\hline & T txt & $\begin{array}{l}\mathrm{T} \\
\text { interp }\end{array}$ & $\begin{array}{l}\mathrm{T} \text { exper } \\
\text { marcado }\end{array}$ & $\begin{array}{l}\text { T exper } \\
\text { no } \\
\text { marcado } \\
\end{array}$ & & $\begin{array}{l}\text { Tema/ } \\
\text { Nuevo }\end{array}$ & Nuevo \\
\hline $\mathrm{a}$ & & & A veces & $\begin{array}{l}\emptyset \\
\text { [nosotros } \\
\text { (Irene y } \\
\text { yo)] }\end{array}$ & $\begin{array}{l}\text { llegamos a } \\
\text { creer }\end{array}$ & & \\
\hline $\mathrm{b}$ & que & & & & era ella & $\begin{array}{l}\text { [[la que no } \\
\text { nos dejó } \\
\text { casarnos]] }\end{array}$ & \\
\hline $\mathrm{c}$ & & & & Irene & $\begin{array}{l}\text { rechazó dos } \\
\text { pretendientes }\end{array}$ & & sin mayor motivo, \\
\hline $\mathrm{d}$ & & & $\underline{\text { a mí }}$ & & se me murió & $\begin{array}{l}\text { Matía } \\
\text { Esther }\end{array}$ & \\
\hline $\mathrm{e}$ & $\begin{array}{l}\text { antes } \\
\text { que }\end{array}$ & & & $\begin{array}{l}\text { Ø [María } \\
\text { Esther y } \\
\text { yo] }\end{array}$ & $\begin{array}{l}\text { llegáramos a } \\
\text { comprome- } \\
\text { ternos. }\end{array}$ & & \\
\hline $\mathrm{f}$ & & & & $\begin{array}{l}\emptyset \\
{[\text { Nosotros }} \\
\text { (Irene y } \\
\text { yo)] }\end{array}$ & $\begin{array}{l}\text { Entramos en } \\
\text { los cuarenta } \\
\text { años }\end{array}$ & & $\begin{array}{lr}\text { con la inexpresada } \\
\text { idea de [[que el } \\
\text { nuestro, simple y } \\
\text { silencioso } & \\
\text { matrimonio r de } \\
\text { hermanos, era } \\
\text { necesaria clausura de } \\
\text { la genealogía } \\
\text { [[asentada por los } \\
\text { bisabuelos en nuestra } \\
\text { casa]] ]]. }\end{array}$ \\
\hline g & & & & $\begin{array}{l}\text { Ø } \\
{[\text { Nosotros }} \\
\text { (Irene y } \\
\text { yo)] } \\
\end{array}$ & $\begin{array}{l}\text { Nos } \\
\text { moriríamos } \\
\text { allí }\end{array}$ & & algún día, \\
\hline $\mathrm{h}$ & & & & $\begin{array}{l}\text { vagos y } \\
\text { esquivos } \\
\text { primos }\end{array}$ & se quedarían & & con la casa \\
\hline $\mathrm{i}$ & $\mathrm{y}$ & & & $\begin{array}{l}\varnothing \text { [vagos y } \\
\text { esquivos } \\
\text { primos] }\end{array}$ & la echarían & & al suelo \\
\hline $\mathrm{j}$ & & & & & & & $\begin{array}{l}\text { para enriquecerse con } \\
\text { el terreno y los } \\
\text { ladrillos; }\end{array}$ \\
\hline $\mathrm{k}$ & & $\begin{array}{l}\mathrm{o} \\
\text { mejor }\end{array}$ & & $\begin{array}{l}\text { nosotros } \\
\text { mismos }\end{array}$ & $\begin{array}{l}\text { la voltearía- } \\
\text { mos }\end{array}$ & & Justicieramen-te \\
\hline 1 & $\begin{array}{l}\text { antes } \\
\text { de } \\
\text { que }\end{array}$ & & & & fuese & & demasiado tarde. \\
\hline
\end{tabular}




\subsection{El sistema de Tema en español}

Todo lo señalado y ejemplificado hasta aquí permite construir el sistema de Tema en español, en lo que concierne al Tema experiencial. Se presenta a continuación (Figura 1) una modificación del sistema elaborado en colaboración con Martin y Quiroz a partir de Moyano (2016) (Martin et al., en prensa, ofrece una versión similar).

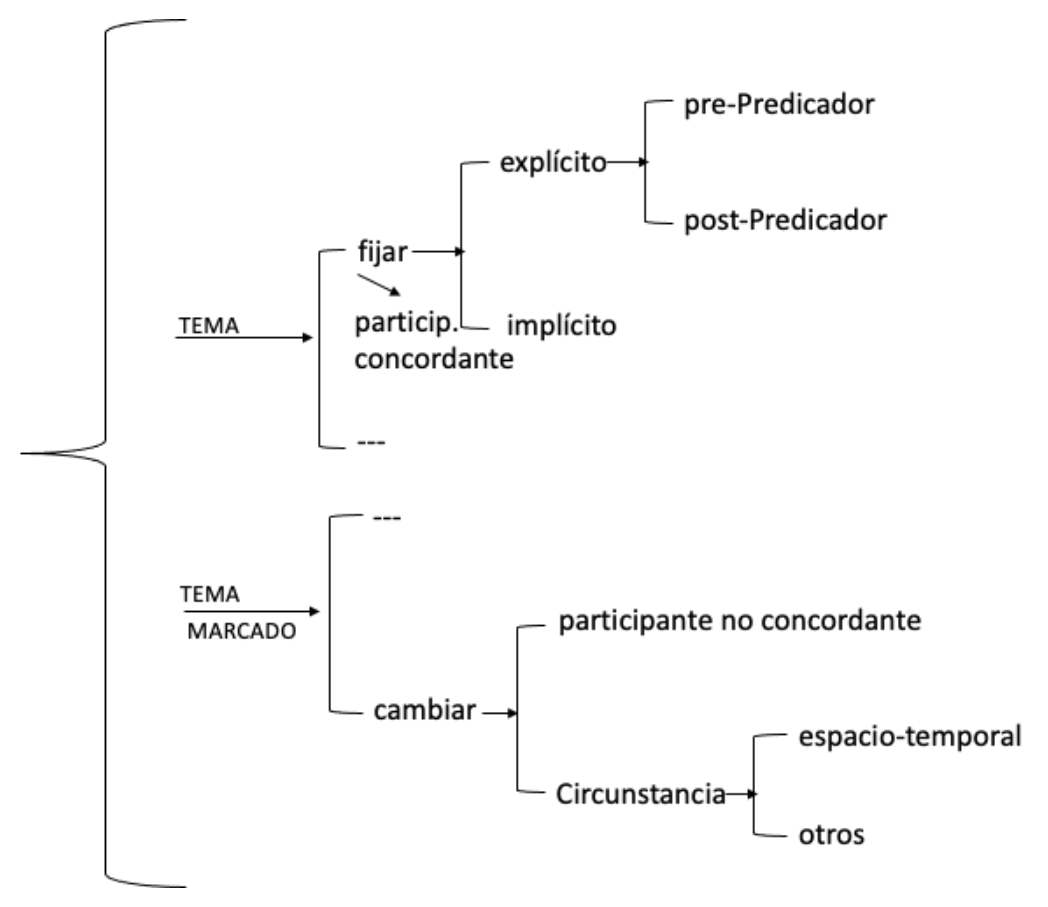

Figura 1. El sistema de Tema experiencial en español.

Como se observa en la Figura 1, se pueden elegir simultáneamente las opciones de Tema y Tema marcado. El Tema, que tiene como función fijar u orientar la mirada del lector/oyente en un aspecto del campo, se realiza siempre mediante un participante concordante con el sufijo verbal, que puede estar explícito o implícito. Si el Tema es explícito, se abren dos opciones: puede ubicarse antes del Predicador (pre-Predicador) o posteriormente a esa función (post-Predicador), en cuyo caso confla con el Nuevo de la cláusula pero aun contribuye a la construcción del método de desarrollo tanto como al punto del texto. Como puede observarse en la primera secuencia de opción, existe en español la posibilidad de que existan cláusulas sin tema, como las que expresan existencia o estado del tiempo (Martin et al., en prensa).

El Tema marcado puede o no estar presente en la cláusula. Cuando está presente, tiene como función cambiar la orientación hacia el campo (Tema 'bisagra', en Moyano, 2010) y puede consistir en un participante no concordante o en una Circunstancia que, a su vez, puede ser temporal o espacial o de otro tipo. 


\section{REFLEXIONES FINALES}

Frente a la controversia acerca de los medios de realización de Tema en español, en el presente artículo se sostiene una posición alternativa a la que han sostenido otros autores en el campo (Taboada, 1995; McCabe, 1999; Montemayor- Borsinger, 2009; Arús, 2010, 2017; Lavid et al., 2010; Gutiérrez, 2011). Para ello, se han tenido en cuenta, en primer lugar, los principios y la metodología propuestos por la tipología lingüística en la LSF, sintetizada en Caffarel et al., 2004a y ejemplificada en diferentes lenguas no solo en ese volumen completo (Caffarel et al., 2004b) sino también en Martin et al. (en prensa). Asimismo, se revisó la definición teórica de la función de Tema en este marco, interpretándola a través de bibliografía relevante. Finalmente, se abordó el análisis de ocho fragmentos de textos de diferentes registros, a fin de lograr definir los medios de realización del Tema en español y poder así graficar una red sistémica de la función en esta lengua.

Como síntesis, cabe finalmente establecer los siguientes puntos:

- El Tema no marcado en español es realizado por concordancia. El sufijo verbal es un marcador de Tema en el rango de la palabra.

- El Tema no marcado en español puede ser explícito o implícito, pero siempre concuerda con el sufijo verbal.

- El Tema en español, cuando es explícito, puede estar antepuesto o postpuesto con respecto al Predicador, y en el segundo caso, confla con el Nuevo.

- El Tema marcado en español se realiza secuencialmente, mediante una Circunstancia (como en muchas otras lenguas) o mediante un participante no concordante, ubicados como primer elemento experiencial de la cláusula. Su función es andamiar un cambio en el discurso.

Como futuras proyecciones para este trabajo, será necesario, en primer lugar definir la realización de Tema en español en cláusulas interrogativas y exclamativas, así como en cláusulas imperativas, estas últimas características del género Instructivo. Asimismo, es necesario revisar si la posición asumida se confirma en otros géneros así como también en registros dialógicos y en registros que presenten el lenguaje como acción (Martin et al, en prensa).

\section{REFERENCIAS BIBLIOGRÁFICAS}

Arús, J. ([2006] 2010). On Theme in English and Spanish: A comparative study. En E. Swain (Ed.), Thresholds and Potentialities of Systemic Functional Linguistics: Multilingual, Multimodal and Other Specialised Discourses (pp. 23-48). Trieste: EUT. 
Arús, J. (2017). Theme in Spanish. En T. Bartlett \& G. O'Grady (Eds.), The Routledge handbook of Systemic Functional Linguistics (pp.194-212). Londres/ Nueva York: Routledge.

Bartlett, T. \& O’Grady, G. (2019). Language characterology and textual dynamics: A crosslinguistic exploration in English and Scottish Gaelic. Special Issue in Communicative Dynamism, Acta Linguistica Hafniensia, 51(1), 124-159.

Caffarel, A., Martin, J. R. \& Matthiessen, C.M.I.M. (2004a). Introduction: Systemic functional typology. En A. Caffarel, J. R. Martin \& C.M.I.M. Matthiessen (Eds.), Language Typology: A Functional Perspective (pp.1-76). Ámsterdam: John Benjamins.

Caffarel, A., Martin, J. R. \& Matthiessen, C.M.I.M. (Eds.) (2004b). Language Typology: A Functional Perspective (pp.1-76). Ámsterdam: John Benjamins.

Cousteau, J. (1994). Las investigaciones geológicas. Enciclopedia del Mar. Barcelona: Folio.

Downing, A. (1991). An alternative approach to Theme: A Systemic-Functional perspective, Word, 40(2), 119-43.

Filice, E. (2010). La estructura temática en el género ensayo: Un análisis sistémicofunctional de escritos de geografía al nivel universitario. Lingüistica Mexicana, $V(1), 111-133$.

Forey, G. \& Sampson, N. (2017). Textual metafunction and Theme. En T. Bartlett \& G. O'Grady (Eds.), The Routledge handbook of Systemic Functional Linguistics (pp. 131-145). Londres/Nueva York: Routledge.

Fries, P. H. (1981). On the status of Theme in English: Arguments from discourse. Forum Linguisticum, 6(1), 1-38.

Gómez González, M. A. \& García Varela, A. P. (2014). Discourse-organizational patterns in English and Spanish. Some notes on the thematic management of news reports. Spanish Journal of Applied Linguistics, 27(1), 87-117.

Gutiérrez, R. M. (2011). Descripción sistémico-funcional y gramática multi-registro. Estudios Filológicos, 47, 59-82.

Halliday, M.A.K. ([1979] 2002). Modes of meanings and modes of expression: Types of grammatical structure and their determination by different semantic function. En J. Webster (Ed.), On Grammar. Collected Works of M.A.K. Halliday (pp.196-218). Londres: Continuum.

Halliday, M.A.K. (1994). An Introduction to Functional Grammar (2nd ed.). Londres: Arnold. 
Halliday, M.A.K. \& Matthiessen, C.M.I.M. (2014). An Introduction to Functional Grammar (4th ed.). Londres: Rouledge.

Halliday, M.A.K. \& McDonald, E. (2004). Metafunctional profile of the grammar of Chinese. En A. Caffarel, J. R. Martin \& C.M.I.M. Matthiessen (Eds.), Language Typology: A Functional Perspective (pp. 305-396). Ámsterdam: John Benjamins.

Lavid, J., Arús, J. \& Zamorano, J. R. (2010). Systemic Functional Grammar of Spanish. A Contrastive Study with English. Londres: Continuum

Martin, J. R. (1983). Participant identification in English. Tagalog and Kate, Australian Journal of Linguistics, 3(1), 45-74.

Martin, J. R. (1992a). Theme, method of development and existentiality: The price of reply. Occasional Papers in Systemic Linguistics, 6, 147-183.

Martin, J. R. (1992b). English Text: System and Structure. Ámsterdam: John Benjamins.

Martin, J. R. (1996). Types of structure: Deconstructing notions of constituency in clause and text. En E. H. Hovy \& D. R. Scott (Eds.), Computational and Conversational Discourse: Burning Issues - an Interdisciplinary Account (pp. 39-66). Heidelberg: Springer, NATO Advanced Science Institute Series F - Computer and Systems Sciences.

Martin, J. R. (2004). Metafunctional profile of the grammar of Tagalog. En A. Caffarel, J. R. Martin \& C.M.I.M. Matthiessen (Eds.), Language Typology: A Functional Perspective (pp. 255-304). Ámsterdam: John Benjamins.

Martin, J. R. (2013). Systemic Functional Grammar: A Next Step into the Theory. Axial Relations. Beijing: Higher Education Press.

Martin J. R. \& Rose, D. (2007). Working with Discourse. Meaning Beyond the Clause (2nd ed.). Londres: Continuum.

Martin, J. R., Matthiessen, C.M.I.M. \& Painter, C. (2010). Deploying Functional Grammar. Beijing: The Commercial Press.

Martin, J. R., Quiroz, B., Wang, P. \& Zhu, Y. (en prensa). Systemic Functional Grammar: Another step into the theory - grammatical description. Beijing: Higher Education Press.

Matthiessen, C.M.I.M. (2004). Descriptive motifs and generalizations. En A. Caffarel, J. R. Martin \& C.M.I.M. Matthiessen (Eds.), Language Typology: A Functional Perspective (pp. 537-662). Ámsterdam: John Benjamins.

Matthiessen, C.M.I.M. \& Halliday, M.A.K. (2009). Systemic functional grammar: A first step into the theory. Beijing: Higher Education Press. 
McCabe, A. (1999). Theme and thematic patterns in Spanish and English history texts. Tesis Doctoral, Aston University, Birmingham, Inglaterra.

Montemayor-Borsinger, A. (2009). Tema. Una Perspectiva Funcional de la Organización del Discurso. Buenos Aires: Eudeba, Enciclopedia Semiológica.

Moya Guijarro, A. J. (2003). Thematic and Topical Structuring in Three Subgenres. A Contrastive Study. Miscelánea, 27, 131-154.

Moyano, E. I. (2010). El sistema de Tema en español: Una mirada discursiva sobre una cuestión controvertida. En M. D. Fernández \& E. Ghio (Eds), El discurso en español y portugués: Estudios desde una perspectiva sistémico-funcional (pp. 39-87). Santa Fe: Universidad Nacional del Litoral.

Moyano, E. I. (2013). Géneros y discurso en los manuales de biología: La construcción del conocimiento y la actividad científica. En E. Moyano (Coord.), Aprender ciencias y humanidades: Una cuestión de lectura y escritura. Aportes para la construcción de un programa de inclusión social a través de la educación lingüística (pp. 229-296). Los Polvorines: UNGS.

Moyano, E. I. (2016). Theme in English and Spanish: Different means of realization for the same textual function. En B. Clark \& J. Arús (Eds.), Special Issue on Communicative Dynamism, English Text Construction, 9(1), 190-220.

Pérez de Cabrera, L. B. (2012). Comparative study of theme functions in Spanish and English academic essays. Científica, 13, 55-70.

Pike, K. L. (1982). Linguistic Concepts: An introduction to Tagmemics. Lincoln: University of Nebraska Press.

Quiroz, B. (2008). Towards a systemic profile of the Spanish mood. Linguistics and the Human Sciences, 4(1), 31-65.

Quiroz, B. (2013). The interpersonal and experiential grammar of Chilean Spanish: Towards a principled Systemic-Functional description based on axial argumentation. Tesis doctoral, University of Sydney, Sydney, Australia.

Quiroz, B. (2015). La cláusula como movimiento interactivo: Una perspectiva semántico-discursiva de la gramática interpersonal del español. DELTA: Documentacão e Estudos em Linguística Teórica e Aplicada, 31(1), 261-301.

Quiroz, B. (2017). The verbal group. En T. Bartlett \& G. O'Grady (Eds.), The Routledge bandbook of Systemic Functional Linguistics (pp. 301-318). Londres/Nueva York: Routledge.

Rose, D. (2001). Some variation in Theme across languages. Functions of Language, 8(1), 109-145. 
Taboada, M. (1995). Theme markedness in English and Spanish: A Systemic-Functional approach. Tesis Doctoral, Universidad Complutense de Madrid, Madrid, España.

Teruya, K. (2004). Metafunctional profile of the grammar of Japanese. En A. Caffarel, J. R. Martin \& C.M.I.M. Matthiessen (Eds.), Language Typology: A Functional Perspective (pp. 185-254). Ámsterdam: John Benjamins.

Thompson, G. \& Thompson, S. (2009). Theme, Subject and the Unfolding of Text. En G. Forey \& G. Thompson (Eds.), Text Type and Texture. In Honour of Flo Davies (pp. 45-69). Londres: Equinox.

\section{REFERENCIA DE TEXTOS ANALIZADOS}

Borges, J. L. (1974). El brujo postergado. Obras Completas (p. 339). Buenos Aires: EMECÉ,

Borges, J. L. (1974). Los dos reyes y los dos laberintos. Obras Completas Tomo 1 (p. 607). Buenos Aires: EMECÉ.

Ciencias Sociales 5. Nación. (2004). Buenos Aires: Puerto de Palos.

Cortázar, J. (2001). Casa tomada. Bestiario. Buenos Aires: Alfaguara.

Quiroga, H. (1984). A la deriva. Cuentos de amor, de locura y de muerte (pp. 60-63). Buenos Aires: Losada.

Scheneiter, O., Fontana, S., Andrés, A. \& Rosso, B. (2006). Efecto del manejo de la defoliación sobre la acumulación de forraje y persistencia de dos cultivares de Trifolium pratense. Revista Argentina de Producción Animal, 26, 11-21.

\section{NOTAS}

${ }^{1}$ Las traducciones de la bibliografía en inglés son propias.

2 Se elige el término 'temática' para traducir topic dado que este término genera otra discusión que se abordará más adelante en este apartado.

${ }^{3}$ En todas las tablas, los elementos marcados en negrita + subrayado corresponden a Tema experiencial marcado; las negritas a Tema experiencial no marcado y la cursiva negrita a Tema/Nuevo. 